\title{
DETERMINATION OF OPTIMAL WORK START TIME
}

\author{
Shu-Guang Li \\ Chang'an University, College of Information Engineering, Xi'an 710064, China \\ E-mails:lxlsg@vip.sina.com,lxlsg@sohu.com
}

Received 20 August 2006; accepted 4 December 2006

\begin{abstract}
The question is: whether the system total travel cost and travel time are reduced by adjusting the work start time or not? This paper proposes the two-level model for answering the question; the upper-level minimizes the system travel cost and travel time by using the work start time as a decision variable, the lower-level models the stochastic dynamic simultaneous route/departure time equilibrium problem. Finally, numerical results of a small network are provided to illustrate the behavior of the model.
\end{abstract}

Keywords: optimal work start time, stochastic dynamic simultaneous route/departure time equilibrium.

\section{Introduction}

In almost all Asian metropolises, as road infrastructure is far below the satisfactory level, we are suffering from heavy road congestion and environmental problems due to exhaust emission. Considering both monetary and space constraints, we cannot expect a large amount of road facilities expansion any more. Therefore, the "Flexible Work Hours (FWH) system" has been introduced recently in order to reduce the peak of commuting demand. FWH is one of the transportation demand management measures to change the distribution of home departure time, as well as that of the work start time.

T. Friesz [1], H.-J. Huang and W. H. K. Lam [2] model the route and departure time choice behavior of travellers by the fixed work start time. C. Hendrickson and G. Kocur [3] propose a model to determine the work start time and the flexibility of the arrival time of employees in order to maximize the employer's benefits. M. Yoshimura and M. Okumura [4] formulate an optimal control model of flexible work hours system under the conditions of system optimization and user equilibrium.

The question arises whether the system total travel cost and travel time are reduced by adjusting the work start time in the view of system manager and travellers or not? The two-level model is used to answer the question. The upper-level minimizes the system travel cost and travel time by using the work start time as a decision variable; the lower-level models the stochastic dynamic simultaneous route/departure time equilibrium problem. The other section is organized as follows. In Section 2, we propose a two-level program model. In Section 3, the heuristic procedure is proposed. Finally, numerical results of a small network are provided to illustrate the behavior of the model.

\section{Model formulation}

Consider a network $G=(N ; A)$, where $N$ is the set of nodes and $A$ is the set of links in the network. Let $R S$ be the set of all $O D$ pairs in the network and $R_{r s}$ be the set of routes between $O D$ pair $r s \in R S$.The studies horizon is discretized into $m$ intervals of length $\delta$ such that $T=m \cdot \delta$. Here, we assume the study horizon is long enough to ensure all travellers can exit from the network after the time $T$. On the other hand, it is also assumed that the value of $\delta$ is small enough so that the discrete time model can approximate its continuous time counterpart.

$f_{p}^{r s}(k)$ - the inflow rate on route $p$ between $O D$ pair rs. $c_{p}^{r s}(k)$ - the actual route travel time experienced on route $p$ between $O D$ pair $r s . c_{p}^{r s}(k)$ - the actual route travel cost experienced on route $p$ between $O D$ pair $r s$. $\hat{c}_{p}^{r s}(k)$ - the perceived route travel cost on route $p$ between $O D$ pair $r s . q^{r s}$ - the demand between $O D$ pair $r s$. $y_{1}$ - the parameter reflected information quality.

The stochastic dynamic route and departure time choice conditions state for each $O D$ pair that the perceived route travel costs for travellers regardless of departure time are equal and minimal. In other words, the perceived route travel costs of any unused route and departure time for each $O D$ pair is greater than or equal to the minimal perceived route travel costs. The stochastic dynamic simultaneous route/departure time user equilibrium condition can be expressed as follows:

$$
f_{p}^{r s}(k)=\frac{\exp \left(-y 1 \cdot c_{p}^{r s}(k)\right)}{\sum_{p} \sum_{k} \exp \left(-y 1 \cdot c_{p}^{r s}(k)\right)} \cdot q^{r s} \quad \forall r s, p, k .
$$



below:

This condition can also be expressed as shown

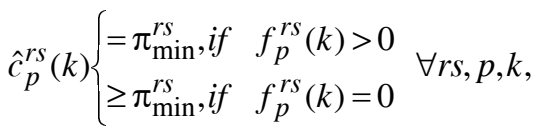

$$
\begin{aligned}
& \pi_{\min }^{r s}=\min \left\{\hat{c}_{p}^{r s}(k): \forall p, k\right\} \text {. }
\end{aligned}
$$

The above expression can further be described by a variational inequality formulation as follows:

$$
\sum_{r s} \sum_{p} \sum_{k} \hat{c}_{p}^{r s^{\prime}}(k) \cdot\left(f_{p}^{r s}(k)-f_{p}^{r s^{\prime}}(k)\right) \geq 0 \quad \forall r s, p, k,
$$

where

$$
\begin{aligned}
& \sum_{p} \sum_{k} f_{p}^{r s}(k) \cdot \delta=q^{r s} . \\
& \hat{c}_{p}^{r s}(k)=c_{p}^{r s}(k)+\frac{1}{y_{1}} \cdot \ln f_{p}^{r s}(k) \quad \forall r s, p, k . \\
& c_{p}^{r s^{*}}(k)=\sum_{a} \sum_{t} c_{a}(t) \cdot \delta_{a p k}^{r s}(t) \quad \forall r s, p, k . \\
& c_{p}^{r s}(k)=\alpha \cdot c_{p}^{r s}{ }^{*}(k)+s c h_{s}(k) \quad \forall r s, p, k .
\end{aligned}
$$

Therefore, the travel cost of a trip from origin $r$ to destination $s$ on path $p$ for a commuter leaving origin at time interval $k$ is:

$$
c_{p}^{r s}(k)=\alpha t_{p}^{r s}(k)+ \begin{cases}\beta\left[t_{s}-\Delta_{s}-k \delta\right] & \text { if } t_{s}-\Delta_{s}>k \delta, \\ \gamma\left[k \delta-t_{s}-\Delta_{s}\right] & \text { if } t_{s}+\Delta_{s}<k \delta,(8) \\ 0 & \text { otherwise }\end{cases}
$$

where: $\alpha$ is a convention factor to transform the path travel time into travel cost. Denote $\left[t_{s}-\Delta_{s}, t_{s}+\Delta_{s}\right]$ as the desired time interval for arrival at the destination $s$ in the network, where: $t_{s}-\Delta_{s}$ is the commuter's desired earliest arrival time, $t_{s}+\Delta_{s}$ is the desired latest arrival time at the destination $s . \beta, \gamma$ is the unit cost of schedule delay (early, late) at the destination $s$. In accordance with the empirical results, we assume that $\gamma>\alpha>\beta$ holds.

At the upper level, the objective is to minimize total system travel time and total travel time by using the work start time as the decision variable.

Total travel cost:

$$
T S C=\sum_{r s} \sum_{p} \sum_{k} f_{p}^{r s}(k) c_{p}^{r s}(k) .
$$

Total travel time:

$$
T T C=\sum_{r s} \sum_{p} \sum_{k} f_{p}^{r s}(k) t_{p}^{r s}(k) .
$$

Total travel cost (total travel time) is obtained by summing the actual travel costs (travel time) of all used paths. This can be expressed as:

$$
\begin{aligned}
& \min J_{t_{s}}=T T C \text { or TSC, } \\
& t_{s \min } \leq t_{s} \leq t_{s \max },
\end{aligned}
$$

where: $\left[t_{s \min }, t_{s \max }\right]$ is the range of work start time.

Here, we use the discrete-time network model proposed by I. Chabini [5].

\section{Algorithm}

The algorithm processes can be depicted as follows: Step 0: Set initial iteration number $t=1$, choose initial path inflow rate $f_{p}^{r s}(k)_{t}$.

Step 1: Perform dynamic network loading, compute $c_{p}^{r s}(k)_{t}$.

Step 2: Calculate an auxiliary path flow $\hat{f}_{p}^{r s}(k)_{t}$ by Equation (1).

Step 3: Move. Find the new path flow pattern $f_{p}^{r s}(k)_{t+1}=f_{p}^{r s}(k)_{t}+\gamma_{t} \cdot\left(\hat{f}_{p}^{r s}(k)_{t}-f_{p}^{r s}(k)_{t}\right)$.

Step 4: Convergence criterion.

If $\sum_{r s} \sum_{i} \frac{\sum_{p k} \sum_{k}\left|f_{p, i}^{r s}(k)_{t+1}-f_{p, i}^{r s}(k)_{t}\right|}{q_{i}^{r s}} \leq \varepsilon$, then stop, otherwise, go to step 1 and set $t=t+1$.

In the algorithm, the step size $\gamma_{t}$ could be determined a priori. For example, the following simplest move size sequences could be applied $\gamma_{t}=1 / t$.

The two-level program problem is transformed to the following two-level unconstrainted minimization:

$$
\begin{aligned}
& \min J_{t_{s}}(f)=T T C \text { or TSC, } \\
& t_{s \min } \leq t_{r s} \leq t_{s \max }, \\
& f \in S\left(t_{r s}\right),
\end{aligned}
$$

where

$$
S\left(t_{r s}\right)=\left\{f \in \Omega\left(t_{r s}\right): c\left(f^{*}-f\right) \geq 0, \forall f^{*} \in \Omega\left(t_{r s}\right)\right\} .
$$

Since the upper level involves only a single decision variable, this two-level problem can be solved by setting $t_{r s}$ as a parameter of the lower problem. We repeatedly have to solve the lower unconstrained minimization by varying value of $t_{r s}$ until we cannot lower the upper level objective.

\section{Numerical example}

In this two-level program model, the control variable is the work start time $t_{\mathrm{s}}$; the parameters are the uncertainty of travel cost $y l$, total travel demand $q^{r s}$, and the flexibility of arrival time $T W$ (these values are exogenous to the program); and the output variables determined by the program are total travel cost TSC and travel time TTC. We use a simple grid network to the idea of using the work start time as a policy instrument to reduce total travel cost TSC or travel time TTC. A simple grid network shown in Fig 1 is used for testing. The network consists of 12 links and 9 nodes, one origin-destination as shown. The four-hour horizon from 6 to 10 a.m is 
divided into 400-time interval of 0.6-minute length. Here we think 6 a.m to be the most early departure time, $\alpha=6.4, \beta=3.9, \gamma=15.21$. We express the travel time function in the following simple form: $c_{a}(k)=\phi_{a}+\lambda_{a} x_{a}(k)$, where $\phi_{a}$ is free flow time in the running segment of link, $\lambda_{a}$ is the parameter. The values of these parameters are given in Table.

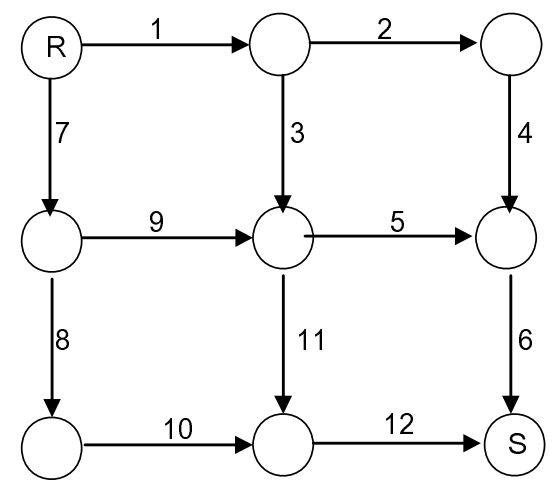

path $1=(1,2,4,6) ; \quad$ path $2=(1,3,5,6)$ path $3=(1,3,11,12) ;$ path $4=(7,9,11,12)$ path $5=(7,9,5,6) ; \quad$ path $6=(7,8,10,12)$

Fig 1. Example network
Parameters of link travel time function

\begin{tabular}{|c|c|c|}
\hline Link & $\Phi_{a}$ & $\lambda_{a}$ \\
\hline 1 & 0.2 & 0.0002 \\
\hline 2 & 0.3 & 0.0004 \\
\hline 3 & 0.2 & 0.00025 \\
\hline 4 & 0.2 & 0.0002 \\
\hline 5 & 0.3 & 0.00045 \\
\hline 6 & 0.2 & 0.00025 \\
\hline 7 & 0.2 & 0.0002 \\
\hline 8 & 0.3 & 0.0004 \\
\hline 9 & 0.2 & 0.00025 \\
\hline 10 & 0.2 & 0.0002 \\
\hline 11 & 0.3 & 0.00045 \\
\hline 12 & 0.2 & 0.00025 \\
\hline
\end{tabular}

We examine the total travel cost and travel time by the varying the values of work start time $t_{\mathrm{s}}$ with different parameter values of $y_{1}, q^{r s}, T W$. For brevity we show only two demand cases in Figs 2, 3. Demands are 20000 and 25000 vehicles. We give the value of flexibility of the arrival time $T W$ in the legend of each graph for each demand level. The total travel cost and travel time cost value with respect to one value of $y 1$ and two values of $T W$ are given in Fig 2, $y_{1}=0.01$. We see the
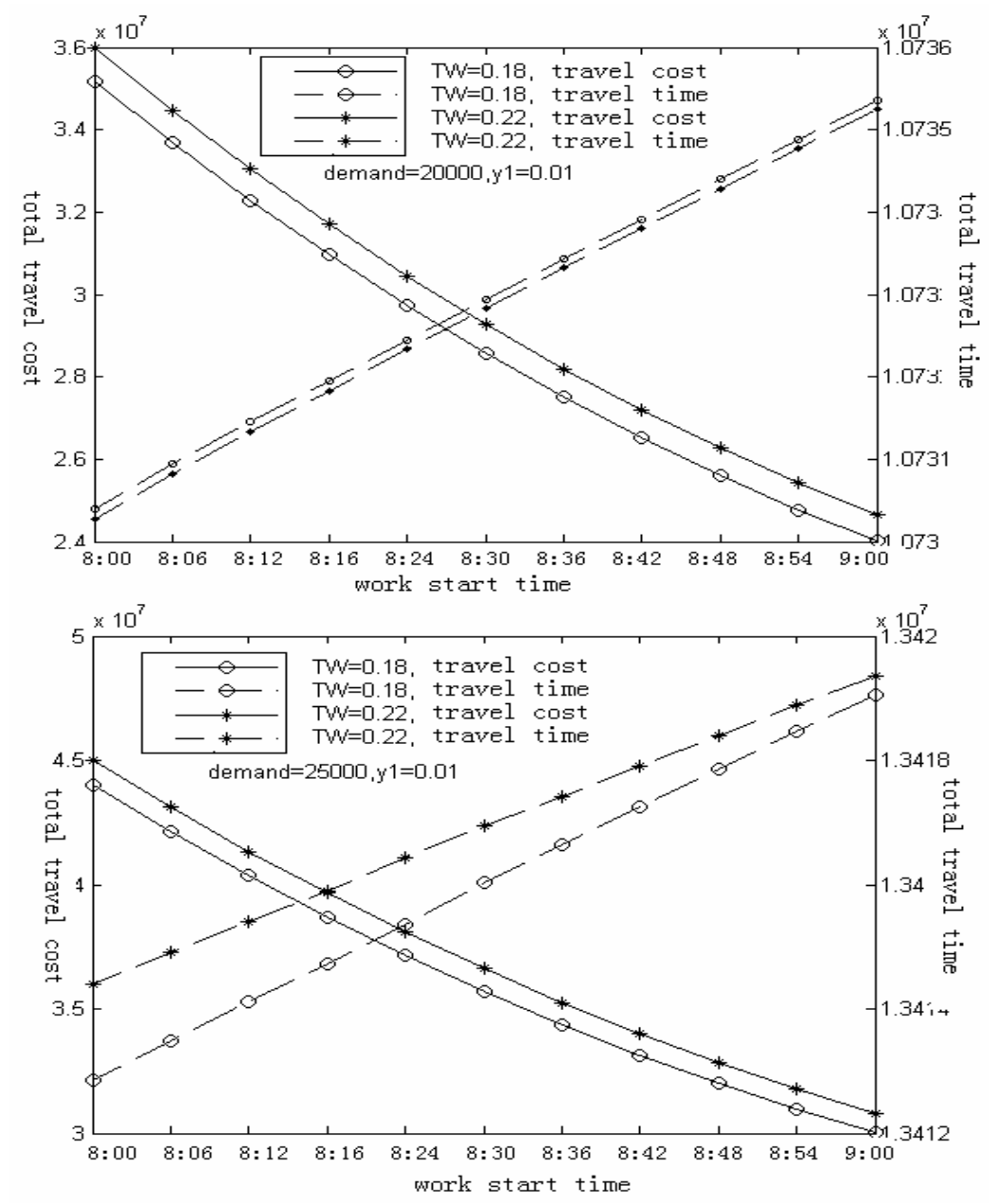

Fig 2. Total system time and cost with respect to work start time 

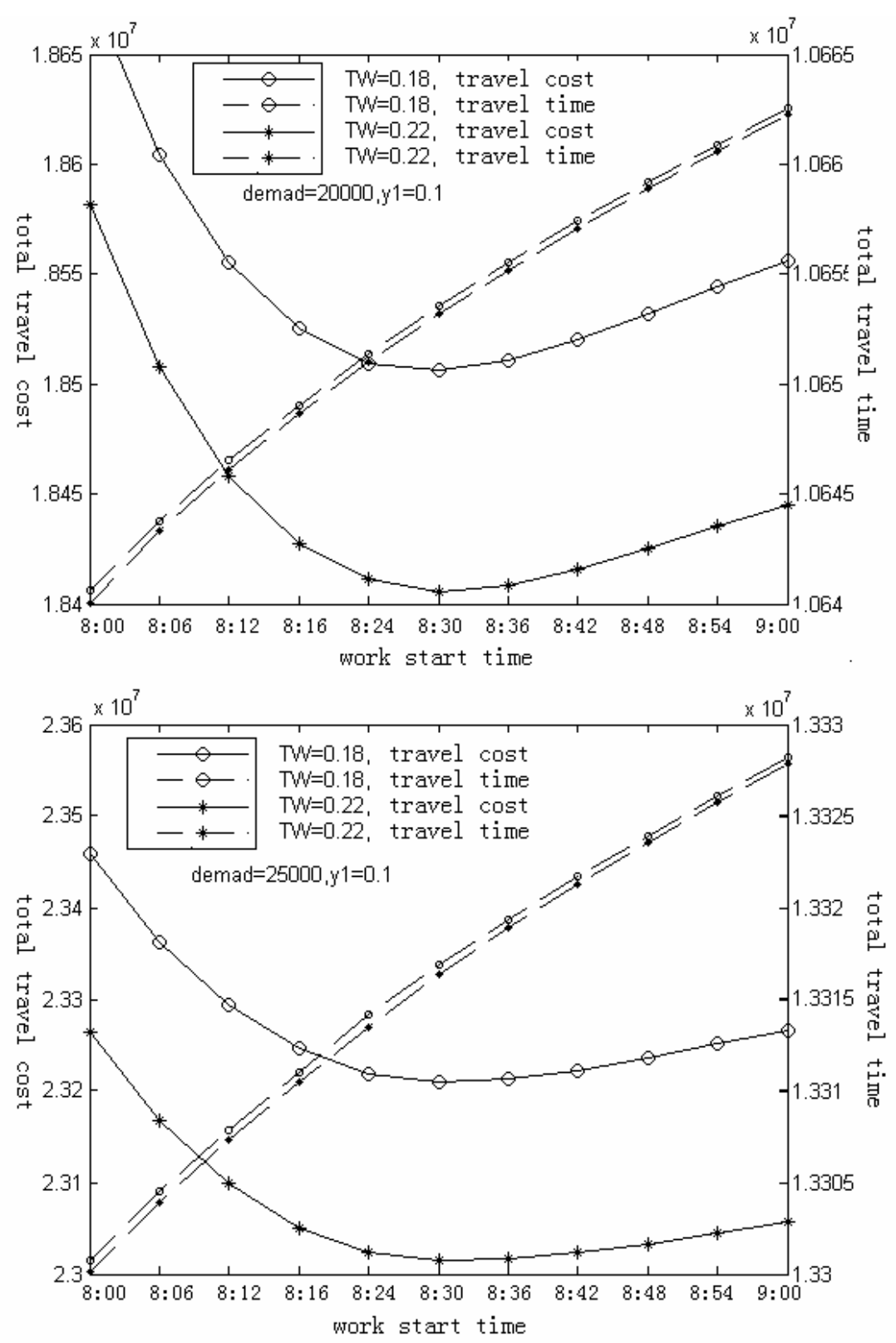

Fig 3. Total system time and cost with respect to work start time

decrease of the total travel cost with the increase of the work start time, as well as the increase of the total travel time with the increase of the work start time. Since the travellers' possibility to be late for work becomes small with the postponement of the work start time, thus the total travel cost is decreased.

In Fig 3, total travel costs do not decrease with the increase of the work start time as $y_{1}=0.01$. When work start time is more than a value, total travel cost is increasing. The change curve of total travel time is similar in Fig 2.

From the change curves of total travel cost and time, we find that the work start time can influence system traffic conditions. In other words, we can improve the traffic conditions by setting the value of the work start time.

\section{Conclusion}

This paper proposes a two-level program model to determine the optimal work start time. The upper level minimizes the total travel cost and travel time by using work start time as a decision variable, the lower level models the dynamic simultaneous route/departure time equilibrium problem. The numerical results, based on a small network, are given. The example shows we can improve the traffic conditions by setting the optimal value of the work start time. In further studies, a detailed simulation is given in actual networks. 


\section{References}

1. FRIESZ, T. L.; BERNSTEIN, D.; SMITH, T. E.; TOBIN, R. L.; WIE, B. W. A variational inequality formulation of the dynamic network user equilibrium problem. Operations Research, 1993, Vol 41, Issue 1, p. 179-191.

2. HUANG, H.-J.; LAM, W. H. K. Modeling and solving the dynamic user equilibrium route and departure time choice problem in network with queues. Transportation Research. Part B: methodological, 2002, Vol 36, No 3, p. 253-273.

3. HENDRICKSON, C.; KOCUR, G. Schedule delay and departure time decisions in a deterministic model. Transportation Science, 1981, Vol 15, p. 62-77.

4. YOSHIMURA, M., OKUMURA, M. Optimal commuting and work start time distribution under flexible work hours system on motor commuting. In Proceedings of the Eastern Asia Society for Transportation Studies, 2001. 10, Vol 3, No 2, p. 455-469.

5. CHABINI, I. Analytical dynamic network loading problem: Formulation, solution algorithms, and computer implementations. Transportation Research Record. Annual Meeting of the Transportation Research Board No 80, Washington, DC, ETATS-UNIS, (07/01/2001) 2001, No 1771, p. 191-200. 